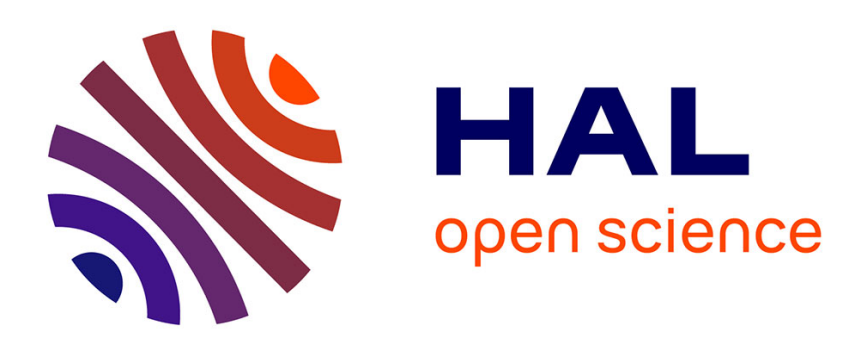

\title{
Etude des ondes de surface permettant la réalisation de cartographies de défauts débouchants
}

\author{
P. Jagnoux, A. Vincent
}

\section{To cite this version:}

P. Jagnoux, A. Vincent. Etude des ondes de surface permettant la réalisation de cartographies de défauts débouchants. Revue de Physique Appliquée, 1988, 23 (8), pp.1383-1396. 10.1051/rphysap:019880023080138300 . jpa-00245960

\section{HAL Id: jpa-00245960 https://hal.science/jpa-00245960}

Submitted on 1 Jan 1988

HAL is a multi-disciplinary open access archive for the deposit and dissemination of scientific research documents, whether they are published or not. The documents may come from teaching and research institutions in France or abroad, or from public or private research centers.
L'archive ouverte pluridisciplinaire HAL, est destinée au dépôt et à la diffusion de documents scientifiques de niveau recherche, publiés ou non, émanant des établissements d'enseignement et de recherche français ou étrangers, des laboratoires publics ou privés. 


\title{
Etude des ondes de surface permettant la réalisation de cartographies de défauts débouchants
}

\author{
P. Jagnoux et A. Vincent $\left(^{*}\right)$ \\ Groupe d'Etudes de Métallurgie Physique et de Physique des Matériaux (ua CNRS 341) INSA de Lyon, \\ bâtiment 502, 69621 Villeurbanne Cedex, France
}

(Reçu le 12 janvier 1988, révisé le 18 avril 1988, accepté le 22 avril 1988)

\begin{abstract}
Résumé. - Nous avons réalisé une étude expérimentale de la propagation d'une onde de surface rayonnante se propageant à une interface liquide-solide et de son interaction avec une fissure idéale débouchant en surface. Les résultats expérimentaux concernant la propagation de cette onde sont comparés aux résultats numériques obtenus en résolvant l'équation de Rayleigh. L'étude se rapportant à l'interaction de l'onde avec le défaut artificiel permet de préciser les propriétés des deux composantes réémises dans le liquide (composante rayonnée par l'onde de surface réfléchie sur l'obstacle et composante diffractée par cet obstacle). Ce travail permet de préciser les propriétés de la cartographie d'une fissure débouchante.
\end{abstract}

\begin{abstract}
Surface acoustic wave (SAW) propagation at a liquid-solid interface and SAW interaction with a surface-breaking idealized crack have been experimentally studied. Experimental results, dealing with propagation, are compared to numerical data got from Rayleigh's equation. The other results, concerning SAW interaction with the defect, allows us to describe the main characteristics of two components reemitted in the liquid (one radiated by the SAW reflected on the obstacle and the other diffracted by the obstacle). The characteristics of a C Scan mapping obtained in the case of a surface-breaking crack are then explained.
\end{abstract}

\section{Introduction.}

Les techniques ultrasonores occupent une place importante en caractérisation et en contrôle non destructif des matériaux. Cette place s'explique notamment par la diversité des mécanismes d'interaction entre les ondes acoustiques et les solides. Ainsi, les ondes ultrasonores permettent de déterminer directement les constantes d'élasticité des matériaux et d'accéder indirectement aux propriétés qui influencent ces constantes d'élasticité dynamiques. Par ailleurs, la diffusion des ondes ultrasonores par la microstructure d'un matériau permet d'en assurer sa caractérisation. Dans le domaine de l'endommagement par fatigue des matériaux, on peut tirer parti des interactions ondes de volume-dislocations, afin de caractériser la mobilité moyenne des dislocations par le suivi de l'atténuation ultrasonore lors des

(*) Egalement au Laboratoire de Traitement du Signal et d'Ultrasons, INSA de Lyon, bâtiment 502, 69621 Villeurbanne Cedex, France. premiers stades de la plasticité cyclique [1] ou de l'endommagement cyclique [2]. Dans le cas où l'endommagement a atteint le stade de la formation de défauts macroscopiques, on peut alors utiliser les divers phénomènes d'interaction onde de volumediscontinuité acoustique, schématisés sur la figure 1, afin de caractériser et de localiser la fissure. Ainsi, outre l'échographie classique utilisant l'onde réfléchie spéculairement sur la fissure, différents auteurs [3-5] ont récemment développé des méthodes utilisant les ondes diffractées par les bords de ces discontinuités acoustiques.

Dans le cas des discontinuités acoustiques débouchant en surface, les ondes de Rayleigh ont été utilisées par différents auteurs, afin de détecter la présence d'une fissure et de mesurer sa profondeur $[6,7]$. Des travaux récents ont montré que l'interaction des ondes de surface rayonnantes se propageant à une interface eau-solide avec des fissures débouchantes ou proches de la surface permet leur détection ainsi que l'évaluation de leur nocivité dans les céramiques [8-10] et dans les métaux [11]. Enfin, on 
doit mentionner le développement de la microscopie acoustique: dans le cas de l'émission-réception, cette technique exploite les phénomènes d'interférences qui se produisent entre l'onde rayonnée par l'onde de surface se propageant à l'interface solideliquide après réflexion sur le défaut débouchant et l'onde réfléchie spéculairement sur la surface de l'échantillon [12].

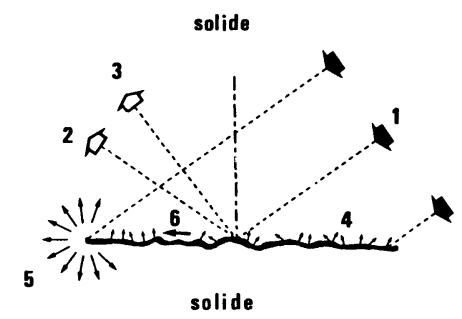

Fig. 1. - Schéma de l'interaction d'une onde longitudinale avec un défaut interne: (1) onde longitudinale incidente ; (2) onde longitudinale réfléchie spéculairement ; (3) onde transversale réfléchie générée par conversion de mode ; (4) ondes de volume rétrodiffusées par la rugosité du défaut ; (5) ondes de volume diffractées par les bords du défaut ; (6) onde de surface générée par conversion de mode.

[Schema of interaction of a compressional wave with an internal defect: (1) incident compressional wave ; (2) reflected compressional wave; (3) reflected transverse wave ; (4) bulk waves scattered by the roughness of the defect; (5) bulk waves diffracted by the edges of the defect; (6) surface wave generated by wave transformation.]

Dans ce contexte, l'objectif essentiel de ce travail est de contribuer à l'étude des ondes de surface rayonnantes et notamment de leur interaction avec les défauts débouchant en surface : en effet, la majorité des travaux cités précédemment font référence à ces mécanismes sans les étudier de façon détaillée. Dans ce but, nous avons choisi des conditions expérimentales (fréquences, matériaux) permettant une bonne caractérisation des ondes de surface. Précisons enfin que cette étude est effectuée en vue de réaliser la cartographie de ces défauts, en travaillant en émission-réception et en immersion. Ainsi, dans la première partie, nous présentons une technique développée récemment au laboratoire [13] qui permet la réalisation de cartographies de défauts débouchant à partir d'un appareillage classique d'imagerie ultrasonore de type $C$ scan (paragraphes 2, 3.1) ; ensuite, à partir des conditions de réalisation de ces cartographies, nous présentons et discutons les expériences fondamentales qui nous permettent de préciser les mécanismes d'interaction onde de surface-défaut mis en jeu (paragraphes 3.2, 4).

\section{Description de l'appareillage.}

Notre appareillage et sa mise en œuvre ayant été décrits par ailleurs [13], nous rappellerons brièvement ses caractéristiques.

Il se compose d'un système programmable d'émission-réception des signaux ultrasonores dont les fonctions programmables sont l'amplitude de l'impulsion d'émission, le gain en réception, ainsi que la position et la largeur d'une porte de sélection électronique. Il permet de numériser, sur huit bits, l'amplitude maximale du signal reçu dans la porte, ainsi que sa position temporelle (sur seize bits).

Ce système d'émission-réception est piloté par un micro-ordinateur Apple IIe qui commande également le déplacement du traducteur suivant deux axes $X$ et $Y$. Ce déplacement est réalisé par l'intermédiaire d'un système de crémaillères et de moteurs pas-à-pas (pas de $10 \mu \mathrm{m}$ ). D'autre part, les réglages de la distance traducteur-pièce et de l'orientation du traducteur sont assurés manuellement par une translation micrométrique au $1 / 100$ de millimètre et par une rotation micrométrique au 1/60 de degré. Les éléments suivants complètent l'appareillage : un oscilloscope Tektronix $2235-100 \mathrm{MHz}$ assure la visualisation des signaux, une table traçante numérique HP 7470A permet le tracé des cartographies. De plus, un oscilloscope numérique Tektronix 2430A peut être mis en œuvre pour la détermination des spectres de fréquence des signaux observés.

Trois traducteurs commerciaux ont été utilisés dans cette étude. Ce sont :

- un traducteur focalisé ponctuellement V312 Panametrics (distance focale $F=25 \mathrm{~mm}$, diamètre de l'élément actif $\varnothing=6 \mathrm{~mm}$, fréquence nominale $f=10 \mathrm{MHz}$ ) ;

- deux traducteurs plans V112 Panametrics ( $\varnothing=6 \mathrm{~mm}, f=10 \mathrm{MHz}$ ).

Rappelons enfin que les cartographies sont construites selon un mode plan présentant les zones isoamplitudes dans le plan de balayage ; l'amplitude en un point est rangée dans la classe $I$, si elle satisfait la relation suivante :

$$
8 \cdot N \cdot(I-1) \leqslant \text { amplitude } \leqslant 8 . N . I .
$$

$N$ est un facteur choisi par l'utilisateur et $I$ est le numéro de la classe. Nous avons limité le nombre de classes à 7 et, à chacune d'entre elles, nous avons associé un caractère alphanumérique dont la densité optique moyenne tend à croître lorsque $I$ augmente de 1 à 7 .

\section{Résultats expérimentaux.}

3.1 CARTOGRAPHIE D'UNE FISSURE DÉBOUCHANTE. - Nous avons déjà indiqué que différents auteurs [8-11], travaillant en émission-réception et 
en immersion avaient pu détecter la présence de fissures débouchantes ou proches de la surface grâce à l'interaction d'une onde de surface rayonnante

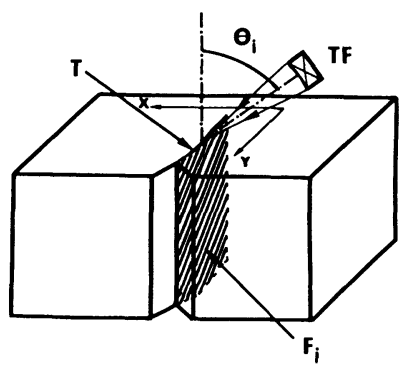

a

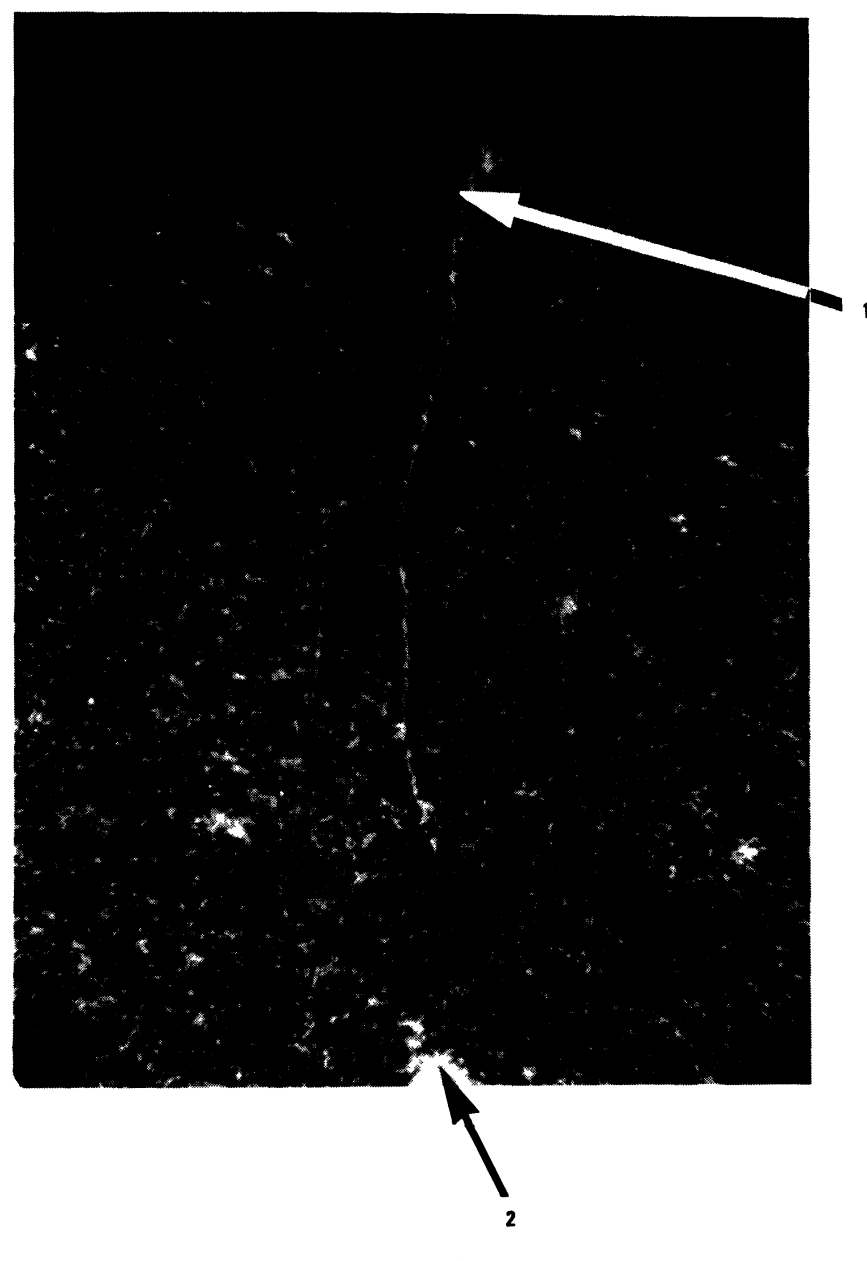

Fig. 2. - Fissure de fatigue développée sur une éprouvette $\mathrm{CT}$ en duralumin: (a) représentation schématique de la disposition des éléments expérimentaux : Fi : fissure, $\mathrm{T}$ : trace de la fissure en surface, $\mathrm{T}_{\mathrm{F}}$ : traducteur focalisé ; (b) photographie optique de la trace en surface: (1) entaille initiale ; (2) trou de référence.

[Fatigue crack in a CT sample made of duraluminum : (a) schematic diagram of the experimental configuration : Fi : crack ; $\mathrm{T}$ : surface trail of crack $; \mathrm{T}_{\mathrm{F}}$ : focused probe ; (b) photograph of the surface crack trail : (1) initial notch ; (2) reference hole.] avec celles-ci. Nous appuyant sur ces travaux, nous avons donc cherché à observer la trace d'une fissure débouchante développée par fatigue dans une éprouvette CT en duralumin 2024. La disposition de cette fissure dans l'éprouvette CT apparaît sur la figure 2a. La longueur de sa trace sur la surface explorée est d'environ $9 \mathrm{~mm}$ : la figure $2 \mathrm{~b}$ représente la photographie optique de cette trace. Dans un premier temps, nous avons mis en évidence un signal associé à cette fissure, avec le capteur focalisé travaillant en émission-réception et en immersion dans les conditions schématisées sur la figure $2 \mathrm{a}$ : nous travaillons sous incidence oblique caractérisée par l'angle d'incidence moyen $\theta_{\mathrm{i}}$ entre la normale à la surface examinée et l'axe du faisceau focalisé ; de plus, le plan d'incidence, défini par l'axe du faisceau focalisé et la normale à la surface, est orienté perpendiculairement à la direction moyenne de la trace de la fissure. Nous avons alors fait varier l'angle d'incidence $\boldsymbol{\theta}_{\mathrm{i}}$ en conservant le centre de la tache focale positionné sur la trace de la fissure et nous avons enfin mesuré l'amplitude du signal provenant de ce point.

La figure 3 présente les résultats obtenus. Nous pouvons observer que l'amplitude du signal présente un maximum pour $\theta_{i}=27^{\circ}$. Lorsque l'on s'écarte de l'incidence $\theta_{\mathrm{i}}$ conduisant à ce maximum, une diminution de l'amplitude précède une augmentation rapide, pour des valeurs de $\theta_{i}$ inférieures à $18^{\circ}$, qui doit être attribuée à la portion du faisceau focalisé subissant une réflexion spéculaire sur la surface de l'éprouvette. Pour $\theta_{i}>27^{\circ}$, l'amplitude décroît et tend vers 0 .

Cette courbe précise donc les meilleures conditions, en émission-réception et avec le capteur focalisé utilisé, pour obtenir un écho pouvant servir

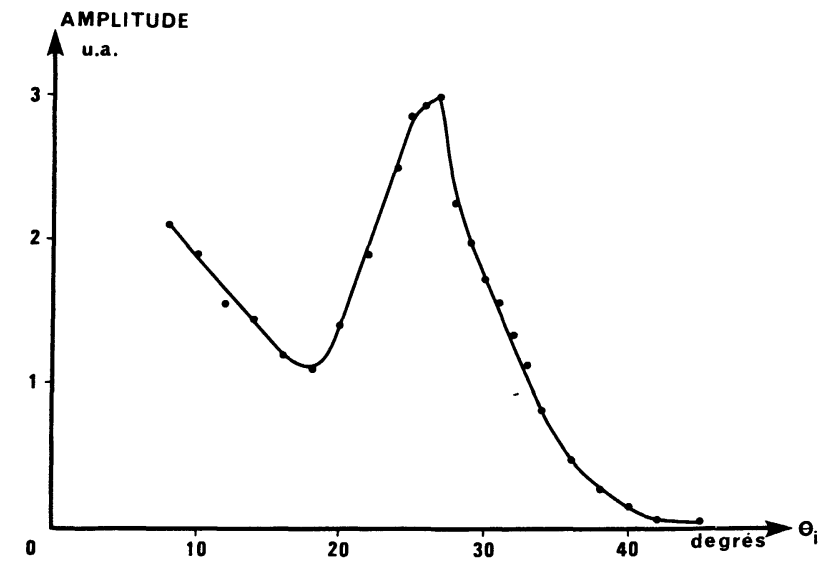

Fig. 3. - Etude angulaire du signal provenant de la fissure en émission-réception: amplitude de l'écho recueilli en fonction de l'orientation $\theta_{i}$ définie sur la figure $2 \mathrm{a}$.

[Angular dependence of the signal amplitude due to the crack in the case of pulse echo technique : wave amplitude versus angle of incidence $\theta_{\mathrm{i}}$ defined in figure 2a.] 
à la détection des défauts de surface dans le duralumin.

Nous avons alors réalisé la cartographie complète de la fissure (Figs. 4a et b). Les conditions de réalisation de cette cartographie sont les suivantes : la tache focale est positionnée sur la surface à examiner; l'angle d'incidence $\theta_{\mathfrak{i}}$, choisi en fonction du résultat précédent, est égal à $27^{\circ}$ et le plan d'incidence est orienté comme précédemment;

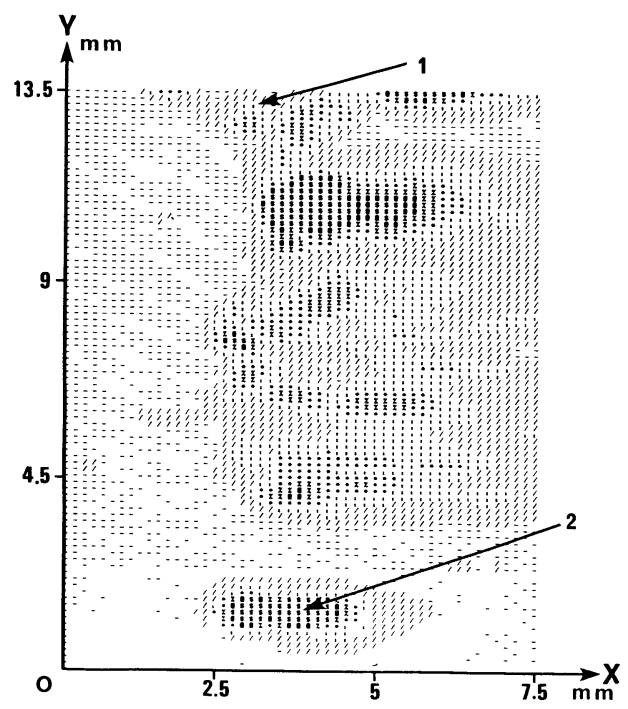

a

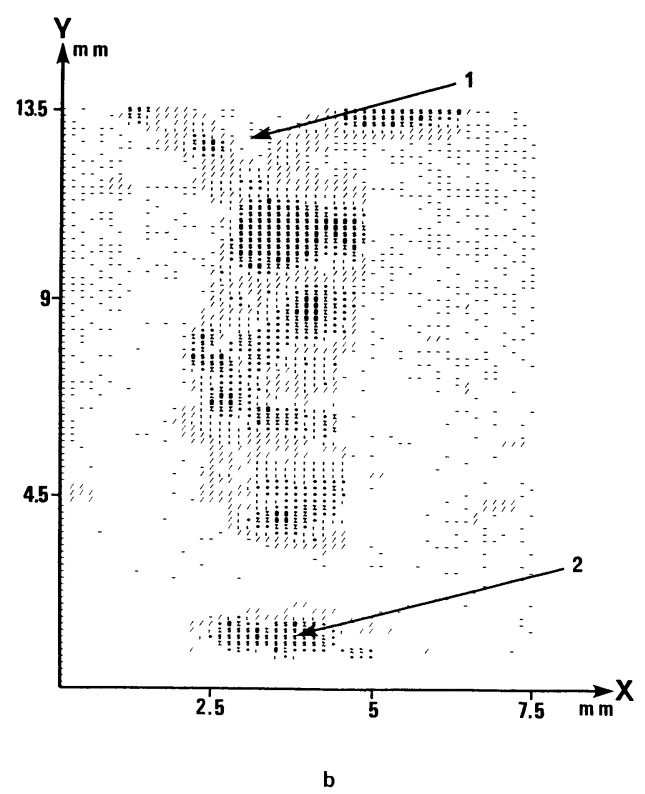

Fig. 4. - Cartographies de la fissure de fatigue en émission-réception avec le traducteur focalisé et $\theta_{\mathrm{i}}=27^{\circ}:$ (1) entaille initiale ; (2) trou de référence. (a) Porte large ; (b) porte étroite.

[C Scan mapping of the crack obtained with the focusing probe at $\theta_{\mathrm{i}}=27^{\circ}:$ (1) initial notch ; (2) reference hole. (a) Wide gate ; (b) narrow gate.] enfin, le balayage $X Y$ est réalisé parallèlement à la surface dont on explore ainsi une zone rectangulaire de dimensions $7,5 \mathrm{~mm} \times 13,5 \mathrm{~mm}$. La première cartographie a été réalisée avec une largeur importante $(\ell=5,1 \mu \mathrm{s})$ de la porte de sélection, dont nous rappelons que le rôle est d'éliminer tous les échos indésirables apparaissant hors de la tranche temporelle intéressante. Cette cartographie montre que le signal est détecté, même à une distance importante de la fissure, ce qui, bien évidemment, limite la résolution de deux défauts proches l'un de l'autre. Heureusement, il est possible d'améliorer cette résolution en diminuant la largeur de la porte (Fig. $4 \mathrm{~b}, \ell=1,2 \mu \mathrm{s}$ ). Par ailleurs, au bas de ces deux cartographies, nous pouvons repérer l'image d'un trou de référence (diamètre $0,6 \mathrm{~mm}$ ) qui a été percé dans le prolongement du plan de fissure, afin de servir de point de repère pour le positionnement du traducteur.

L'examen de ces cartographies confirme l'interprétation des observations précédemment citées en référence [8-11]. C'est à dire que l'écho détecté ne peut être dû à une interaction directe de l'onde incidente avec le bord de la fissure. En effet la largeur apparente de la fissure, de l'ordre de $5 \mathrm{~mm}$, sur la cartographie de la figure $4 a$, étant bien supérieure au diamètre de la tache focale (de l'ordre de $0,6 \mathrm{~mm}$ à $-6 \mathrm{~dB}$ ), cette cartographie montre qu'il existe un signal même lorsque le faisceau frappe à côté de la trace externe de la fissure. Nous sommes donc conduits à tenir compte des phénomènes de conversion de mode. A partir des valeurs des vitesses des ondes ultrasonores dans l'eau et le duralumin donnée par Handbook of Chemistry and Physics [14], nous avons calculé les angles critiques des ondes longitudinales et transversales à une interface eau-duralumin; nous avons également déterminé la valeur de l'angle de Rayleigh à partir du calcul de la vitesse d'une onde de surface à une interface eau-duralumin (cf. paragraphe 4.1.1). Nous trouvons respectivement :

$$
\theta_{\mathrm{CL}}=13^{\circ} 43^{\prime}, \theta_{\mathrm{CT}}=28^{\circ} 36^{\prime} \text { et } \theta_{\mathrm{R}}=30^{\circ} 43^{\prime} \text {. }
$$

Compte tenu de la valeur de l'angle d'incidence $\theta_{\mathrm{i}}$ utilisé et de l'angle d'ouverture du faisceau focalisé $\alpha \approx 14^{\circ}$, évalué à partir de la relation géométrique $\operatorname{tg}(\alpha / 2) \approx \varnothing / 2 F$, nous pouvons en déduire que deux types d'ondes sont générés dans le matériau et peuvent interagir avec le défaut :

- des ondes transversales obliques ;

- une onde de surface.

Le plan de la fissure étant orienté quasiment perpendiculairement à la surface de l'échantillon, la direction de la réflexion spéculaire sur cet obstacle ne peut pas coïncider avec la direction d'incidence et ce mécanisme ne peut pas contribuer de façon significative au phénomène observé. La suite de 
l'étude expérimentale s'attache donc à caractériser la propagation des ondes de surface et leur interaction avec un obstacle acoustique.

3.2 ETUdE DES ONDES DE SURFACE. - Afin de réaliser la partie expérimentale de cette étude dans les meilleures conditions, nous avons dû travailler avec un autre couple métal-eau que le couple duralumin-eau, car, aux fréquences mises en œuvre dans ce travail, les ondes de surface s'atténuent très rapidement à une telle interface. En conséquence, nous avons réalisé l'étude des ondes de surface se propageant à une interface acier-eau ; en effet, dans ce cas, l'onde de surface est environ trois fois moins atténuée que dans le cas du couple duralumin-eau pour un parcours de $3 \mathrm{~mm}$.

La valeur de $\theta_{R}$, pour l'acier choisi (A60), est égale à $30^{\circ} 7^{\prime}$ et la vitesse des ondes de surface est $C_{\mathrm{R}}=2989 \mathrm{~m} \mathrm{~s}^{-1}$. Afin de réduire au maximum l'ouverture angulaire des faisceaux, nous avons travaillé avec les deux traducteurs plans (dont les caractéristiques ont été données au paragraphe 2), l'un étant utilisé en émetteur, l'autre en récepteur. L'angle d'incidence de l'émetteur est réglé égal à $\theta_{\mathrm{R}}$; le récepteur est fixé sur un plateau goniométrique monté sur la tête de la table à déplacements croisés.

3.2.1 Onde diffusée vers l'avant. - Lors de la propagation d'une onde de surface, la réémission dans le liquide d'une partie de l'énergie de cette onde est un phénomène bien connu $[10,11]$. De toute évidence, cette réémission contribue à l'atténuation de l'onde lors de son parcours. Nous avons, en premier lieu, réalisé une étude angulaire de ce champ réémis en faisant varier l'angle de réception $\theta_{r}$, le traducteur-récepteur visant toujours le même point de la surface de l'échantillon (Fig. 5a) ; précisons qu'afin d'éviter toute interférence avec le faisceau réfléchi spéculairement, ce point visé est situé en dehors de cette zone de réflexion spéculaire : dans le cas de l'expérience présentée sur la figure 5, le décalage entre le point d'intersection de l'axe du faisceau incident avec l'interface eau-solide et le point visé a été fixé à $X=18 \mathrm{~mm}$ (cf. Fig. 5a).

La figure $5 \mathrm{~b}$ montre qu'un maximum de réémission est détecté pour $\theta_{\mathrm{r}}$ égal à $\theta_{\mathrm{R}}$.

3.2.2 Application à l'étude de l'atténuation de l'onde de surface. - Nous avons réalisé une évaluation de l'atténuation de l'onde de surface en mesurant l'amplitude de l'onde réémise vers l'avant en différents points (espacés d'une distance égale à $1 \mathrm{~mm}$ ) du trajet suivi par l'onde de surface. Précisons que la région particulière correspondant à la réflexion spéculaire a également été évitée dans cette expérience : avec l'hypothèse de faisceaux incident et réfléchi cylindriques cette réflexion spéculaire peut être détectée par le récepteur de $X=-X_{0}$ à
$X=X_{0}$ avec $X_{0}=7 \mathrm{~mm}$, dans le cas de l'incidence $\theta_{i}=30^{\circ} 7^{\prime}$ (Fig. 5a) et avec l'angle de réception réglé de façon à correspondre au maximum de réémission, c'est-à-dire $30^{\circ} 7^{\prime}$. L'amplitude détectée
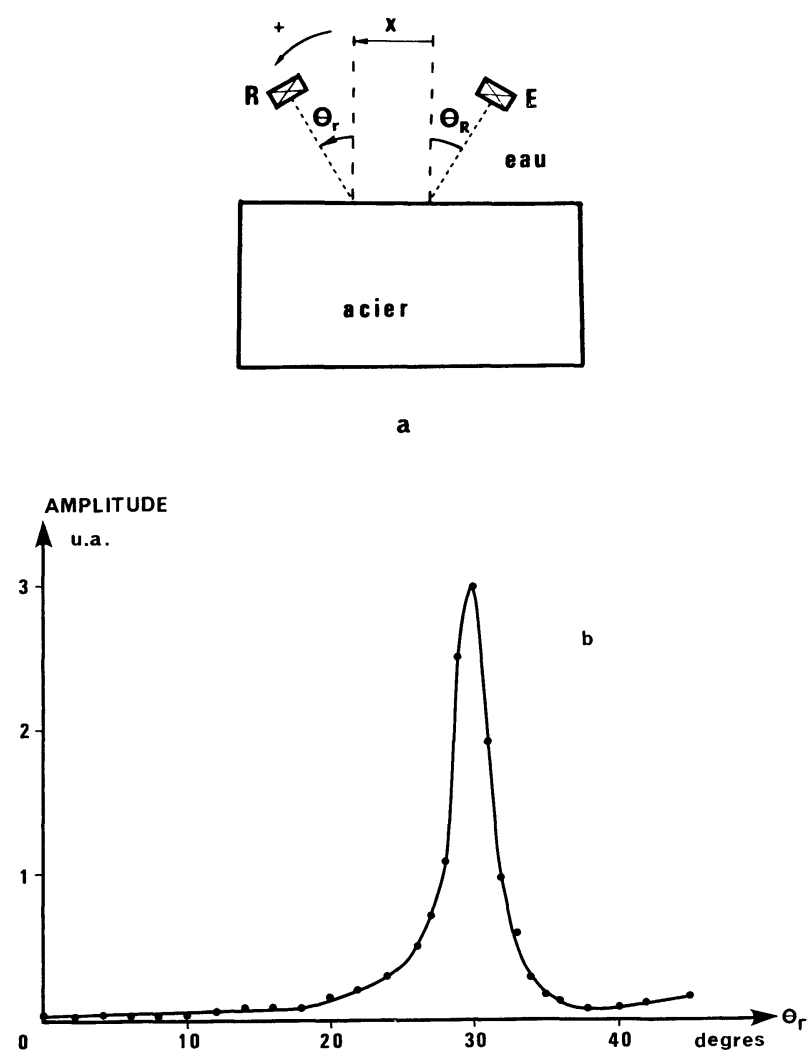

Fig. 5. - Etude angulaire du champ rayonné vers l'avant par une onde de surface se propageant à une interface eauacier : (a) procédure expérimentale : $\mathrm{E}:$ traducteur plan émetteur ; $\mathrm{R}$ : traducteur plan récepteur ; $X=18 \mathrm{~mm}$; (b) amplitude de l'écho détecté en fonction de la direction d'observation $\theta_{\mathrm{r}}$.

[Angular dependence of the wave radiated by a surface wave at a steel-water boundary: (a) experimental configuration : $\mathrm{E}$ : emitting plane probe $; \mathrm{R}$ : receiving plane probe $; X=18 \mathrm{~mm}$; (b) wave amplitude versus $\theta_{\mathrm{r}}$.]

décroît très rapidement lorsque la distance parcourue par l'onde augmente (Fig. 6). De plus, la figure 7 présente trois spectres $(b, c, d)$ de signaux sélectionnés parmi l'ensemble de ceux qui ont été numérisés sur le parcours étudié. Nous donnons également en référence (a) le spectre du signal obtenu en transmission avec les deux capteurs plans positionnés l'un en face de l'autre et séparés d'une distance conduisant à un temps de parcours de l'onde détectée égal à celui mesuré pour les signaux précédents $(\approx 40 \mu \mathrm{s})$. On peut remarquer que les composantes hautes fréquences $(>10 \mathrm{MHz})$ ont pratiquement disparu après un parcours $X-X_{0}$ égal à $12 \mathrm{~mm}$. 


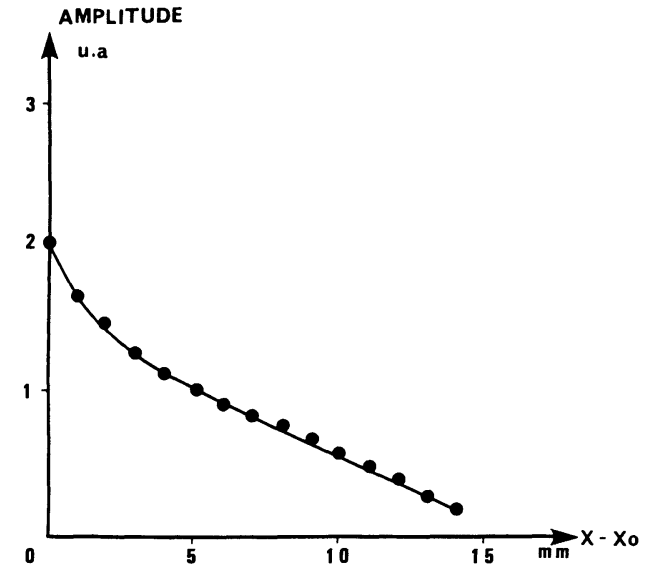

Fig. 6. - Mesure de l'atténuation d'une onde de surface se propageant à une interface eau-acier : amplitude de l'onde rayonnée à l'angle $\theta_{\mathrm{R}}$ en fonction du chemin $X-X_{0}$ parcouru par l'onde de surface. (L'origine $X_{0}$ est située hors de la zone de réflexion spéculaire.)

[Attenuation of the surface wave at a steel-water boundary : amplitude of the radiated wave measured at $\theta_{\mathrm{r}}$ versus distance $X-X_{0}$ travelled by the surface wave (the origin $X_{0}$ is located out of the reflected wave region).]

3.2.3 Interaction d'une onde de surface avec un défaut modèle.

3.2.3.1 Réalisation d'un modèle. - Afin de s'affranchir de la géométrie de la fissure réelle, nous avons modélisé celle-ci par deux demi-plans parallèles séparés de quelques centièmes de millimètre. Ce modèle est facilement réalisable à l'aide de deux blocs parallélépipédiques séparés par une cale d'épaisseur et maintenus dans un étau. Ces blocs ont été réalisés avec l'acier décrit précédemment et nous avons utilisé une cale d'épaisseur de $4 / 100$ de millimètre.

Précisons enfin que l'assemblage étant réalisé en immersion, notre fissure modèle contient donc de l'eau.

3.2.3.2 Etude angulaire de l'onde détectée. - Le traducteur-émetteur est réglé à l'angle de Rayleigh $\left(\theta_{\mathrm{R}}=30^{\circ} 7^{\prime}\right)$ et nous faisons varier l'angle de réception $\theta_{\mathrm{r}}$ entre -45 et $+35^{\circ}$ (Fig. 8a) ; le traducteurrécepteur est positionné de façon à viser toujours le même point de l'arête du défaut modèle ; dans la suite, cette position sera repérée par l'abscisse $X=0$, l'axe $\mathrm{O} X$ étant la direction de déplacement $\mathrm{du}$ traducteur perpendiculaire à l'arête du défaut (Fig. 8a). De plus, la distance récepteur-arête est maintenue constante et égale à $30 \mathrm{~mm}$.

La figure $8 \mathrm{~b}$, obtenue dans ces conditions, montre que l'amplitude maximale détectée sur le récepteur présente deux maxima pour $\theta_{\mathrm{r}}=\theta_{\mathrm{R}}$ et $\theta_{\mathrm{r}}=-\theta_{\mathrm{R}}$.

Nous avons ensuite cherché à déterminer le rôle joué par chaque arête dans le phénomène observé.
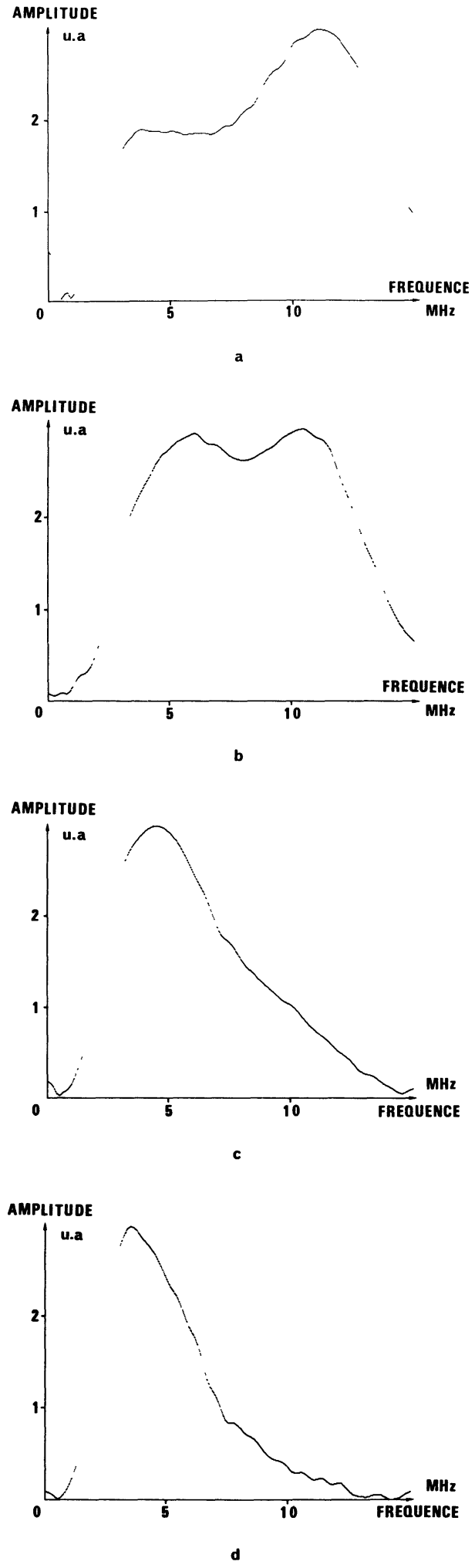

Fig. 7. - Etude spectrale du signal rayonné vers l'avant en différents points du parcours de l'onde de surface : (a) spectre obtenu en transmission, les deux capteurs se faisant face ; (b) $X-X_{0}=0 \mathrm{~mm}$; (c) $X-X_{0}=6 \mathrm{~mm}$; (d) $X-X_{0}=12 \mathrm{~mm}$.

[Frequency spectra of radiated waves versus $x-x_{0}$ : (a) spectrum obtained the probes facing each other; (b) $X-X_{0}=0 \mathrm{~mm}$; (c) $X-X_{0}=6 \mathrm{~mm}$; (d) $X-X_{0}=$ $12 \mathrm{~mm}$. 

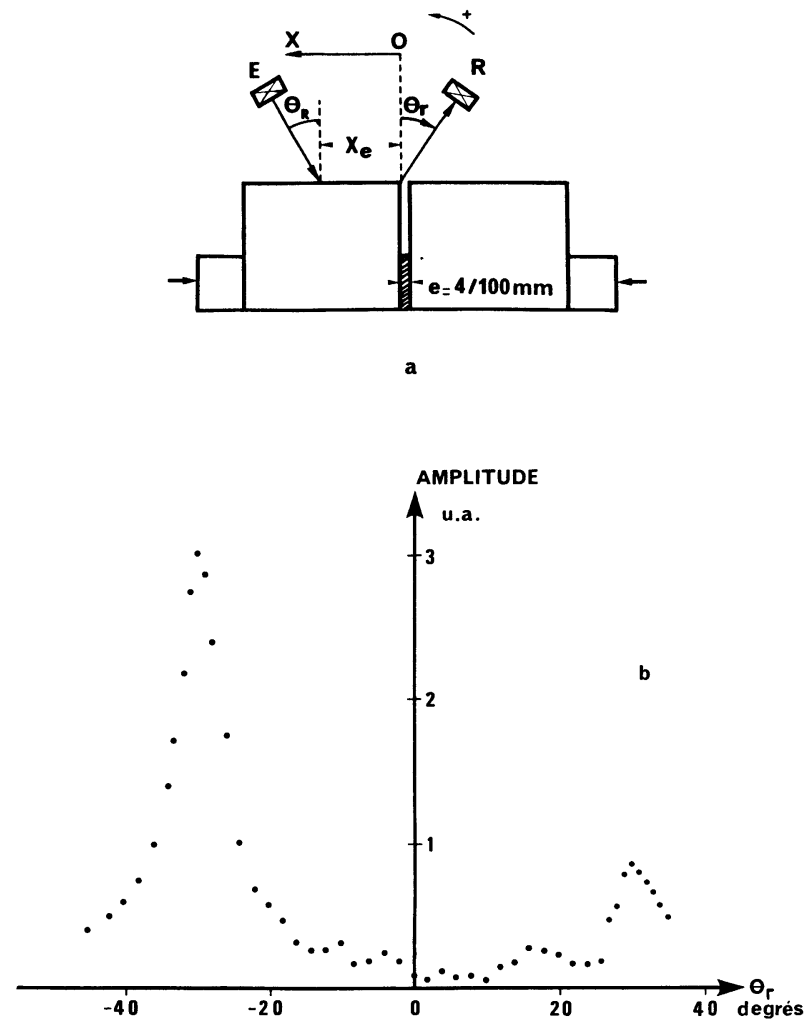

Fig. 8. - Etude de l'interaction d'une onde de surface avec une fissure modèle: (a) réalisation de la fissure modèle et situation des traducteurs : $\mathrm{E}$ : traducteur émet-

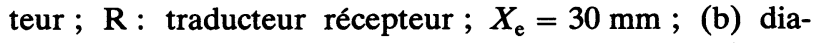
gramme angulaire de réception : amplitude $=f\left(\theta_{\mathrm{r}}\right)$.

[Surface wave interaction with an idealized crack: (a) experimental configuration; $\mathrm{E}$ : emitting probe ; $\mathrm{R}$ : receiving probe ; $X_{\mathrm{e}}=30 \mathrm{~mm}$; (b) wave amplitude versus $\theta_{\mathrm{r}}$ ]

Pour ce faire, nous avons réalisé la même expérience que précédemment en ôtant un bloc (Fig. 9a). La courbe obtenue (Fig. 9b) est semblable d'allure à celle obtenue avec les deux blocs et leurs amplitudes diffèrent seulement d'environ $1 \mathrm{~dB}$. Il semble donc que le phénomène observé soit principalement produit lors de l'interaction de l'onde de surface avec la première arête. Le défaut modèle peut donc être simplifié à la première interface limitant la propagation de l'onde de surface.

Une étude fine des signaux reçus montre qu'en fait l'amplitude maximale détectée peut provenir de différents signaux. Dans le cas du défaut modèle simplifié, nous avons pu observer les situations suivantes en fonction de l'angle $\theta_{\mathrm{r}}$ (le défaut modèle complet conduit à des observations similaires) :

\section{- domaine a :}

pour $-45^{\circ} \leqslant \theta_{\mathrm{r}} \leqslant-36^{\circ}$, il apparaît deux signaux distincts temporellement. Le premier signal reçu, que nous appellerons Ia, présente une position temporelle fixe, quel que soit l'angle $\theta_{\mathrm{r}}$. Le second
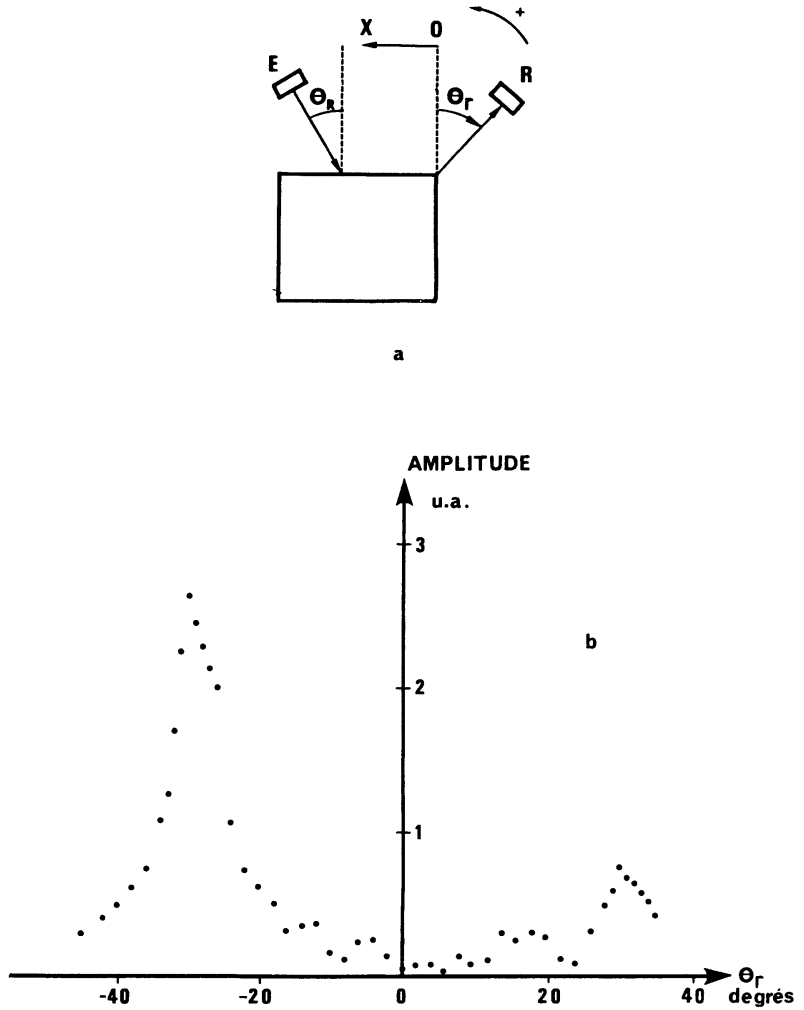

Fig. 9. - Etude de l'interaction d'une onde de surface avec un défaut simplifié : (a) procédure expérimentale : $\mathrm{E}$ : traducteur émetteur; $\mathrm{R}$ : traducteur récepteur ; (b) diagramme angulaire de réception : amplitude $=f\left(\theta_{\mathrm{r}}\right)$.

[Surface wave interaction with a corner : (a) experimental configuration : $\mathrm{E}$ : emitting probe ; $\mathrm{R}$ : receiving probe ; (b) wave amplitude versus $\theta_{\mathrm{r}}$.]

signal, nommé IIa, se rapproche du signal Ia lorsque $\theta_{\mathrm{r}}$ varie de $-45^{\circ}$ à $-36^{\circ}$ et il se confond avec celui-ci lorsque $\theta_{\mathrm{r}}$ dépasse $-35^{\circ}$ environ. De plus, l'amplitude de Ia est supérieure à celle de IIa sauf vers la limite supérieure du domaine où cette situation tend à s'inverser.

Par ailleurs, lorsque le récepteur se déplace perpendiculairement à l'arête du bloc, suivant la direction $\mathrm{O} X$, Ia présente une amplitude maximale au voisinage de $X=0$; au contraire, l'amplitude de IIa augmente graduellement lorsque $X$ croît.

Les courbes Ia et IIa (Fig. 10a) présentent ainsi l'évolution de l'amplitude des signaux Ia et IIa, pour $\theta_{\mathrm{r}}=-38^{\circ}$, en fonction de la position $X$ du récepteur.

La figure 11 présente un oscillogramme réalisé pour ce même angle et pour la position $X=$ $5 \mathrm{~mm}$ : cet oscillogramme est représentatif de ce qui est observé dans toute la région $4<X<8 \mathrm{~mm}$ pour laquelle, comme le montre la figure $10 \mathrm{a}$, les signaux Ia et IIa présentent alors des amplitudes comparables et ils sont donc observables simultanément et distinctement dans de bonnes conditions. 

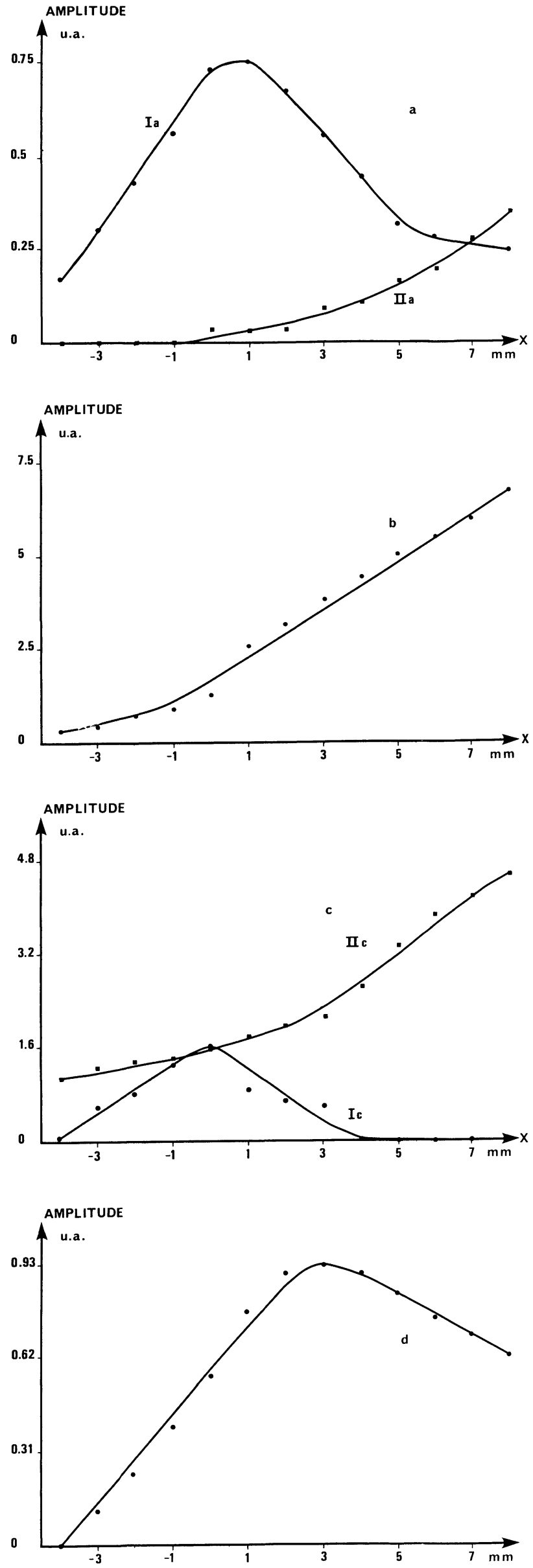

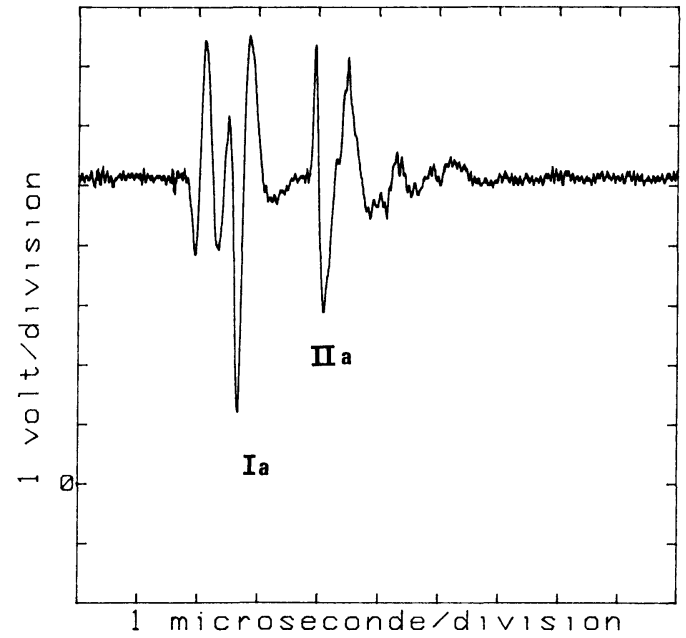

Fig. 11. - Oscillogramme observé pour $\theta_{\mathrm{r}}=-38^{\circ}$ et $x=5 \mathrm{~mm}$ dans les mêmes conditions que la figure $9 \mathrm{a}$.

[Oscilloscope tracing of the signal observed for $\theta_{\mathrm{r}}=$ $-38^{\circ}$ and $x=5 \mathrm{~mm}$ in same experimental conditions as figure 9a.]

\section{- Domaine b :}

pour $-35^{\circ} \leqslant \theta_{\mathrm{r}} \leqslant-24^{\circ}$, les deux signaux sont confondus. Il n'apparaît pas de maximum pour l'amplitude du signal global lorsque le traducteur récepteur est déplacé suivant $\mathrm{O} x$ comme le montre la courbe II'b (Fig. 10b) réalisée pour $\theta_{\mathrm{r}}=-30^{\circ}$. Cette situation n'est pas étonnante si l'on considère qu'en extrapolant les résultats du domaine a, pour lequel l'amplitude de IIa tendait à dépasser celle de Ia vers la limite supérieure du domaine, le signal II est ici devenu la composante principale du signal global.

\section{- Domaine c :}

pour $-22^{\circ} \leqslant \theta_{\mathrm{r}} \leqslant-10^{\circ}$, deux signaux sont à nouveau discernables, mais c'est maintenant le deuxième signal reçu qui possède une position temporelle fixe en fonction de $\theta_{\mathrm{r}}$. Son amplitude présente un maximum suivant $O X$ (courbe Ic, Fig. 10c). Par contre, l'amplitude du premier signal reçu, maintenant noté IIc en raison de la similitude

Fig. 10. - Evolution de l'amplitude des signaux détectés en fonction du déplacement latéral $x$ de la tache focale du récepteur par rapport à l'arête de la discontinuité (cf. Fig. 9a) : (a) $\theta_{\mathrm{r}}=-38^{\circ}$; (b) $\theta_{\mathrm{r}}=-30^{\circ}$; (c) $\theta_{\mathrm{r}}=-16^{\circ}$; (d) $\theta_{\mathrm{r}}=30^{\circ}$.

[Evolution of the amplitude of the different detected waves versus the lateral shift $x$ of the focal spot (cf. Fig. 9a) : (a) $\theta_{\mathrm{r}}=-38^{\circ}$; (b) $\theta_{\mathrm{r}}=-30^{\circ}$; (c) $\theta_{\mathrm{r}}=-16^{\circ}$; (d) $\theta_{\mathrm{r}}=30^{\circ}$.] 
de ses propriétés avec celles de IIa, augmente progressivement suivant cette direction (courbe IIc, Fig. 10c); de plus, pour un point fixe visé sur la surface $(X>0)$, cette amplitude diminue lorsque l'angle de réception se rapproche de la normale à l'interface acier-eau.

\section{- Domaine d:}

enfin, pour $-8^{\circ} \leqslant \theta_{\mathrm{r}} \leqslant 35^{\circ}$, nous n'avons observé qu'un seul signal. Pour un angle $\theta_{\mathrm{r}}$ constant, l'amplitude de ce signal, noté IId, présente un maximum en fonction de $X$, au voisinage de $X=3 \mathrm{~mm}$. Ceci peut être observé sur la figure $10 \mathrm{~d}$ pour $\theta_{\mathrm{r}}=30^{\circ}$.

3.2.3.3 Influence de l'orientation de l'arête. - Ce paramètre n'a pas été étudié avec l'aide des traducteurs plans. Nous avons travaillé en émission-réception avec le traducteur focalisé et nous nous sommes placés dans les conditions de détection optimale : angle d'émission-réception $\theta_{i}=27^{\circ}$ et point de focalisation réglé sur l'arête du bloc. Nous avons alors mesuré l'amplitude du signal détecté pour différentes orientations $\beta$ du plan d'incidence contenant l'axe $\mathrm{du}$ faisceau par rapport au plan perpendiculaire à l'arête du coin. Dans ces conditions, l'amplitude est maximale pour $\beta=0$, c'est-à-dire lorsque l'onde de surface arrive perpendiculairement à l'arête du défaut modèle (Fig. 12) ; elle a chuté de $6 \mathrm{~dB}$ pour une désorientation de l'ordre de $7^{\circ} 30^{\prime}$.

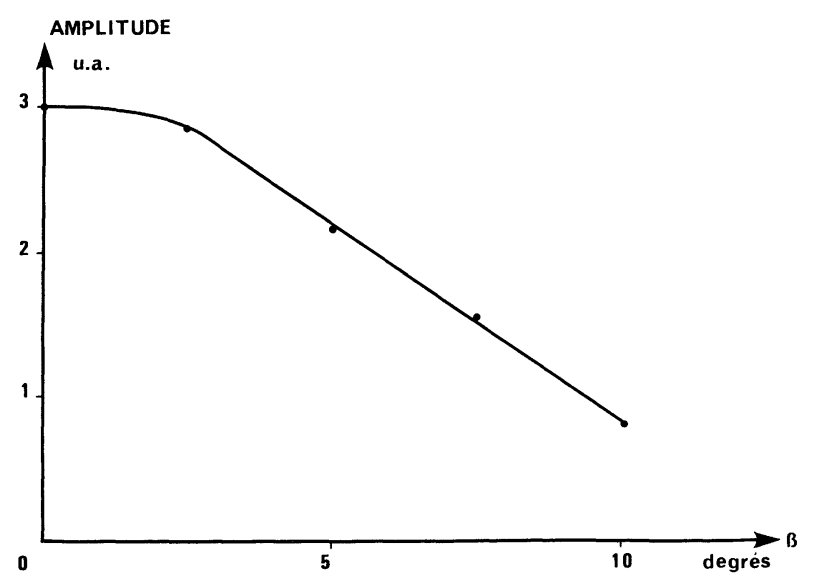

Fig. 12. - Variations de l'amplitude du signal détecté en fonction de l'orientation $\beta$ du plan d'incidence de l'axe du faisceau par rapport au plan perpendiculaire à l'arête du coin.

[Wave amplitude versus the angle $\beta$ between the plane of incidence of the beam axis and the plane perpendicular to the crack edge.]

\section{Discussion.}

Cette discussion comprend deux parties principales. La première concerne la propagation des ondes de surface et leur interaction avec le défaut modèle.
Dans la deuxième partie, avec l'aide des conclusions de la première, nous discutons les phénomènes permettant la réalisation des cartographies de la fissure réelle.

\subsection{LES ONDES DE SURFACES.}

4.1.1 Propagation. - La propagation des ondes de surface a été étudiée théoriquement et expérimentalement depuis de nombreuses années [15, 16]. La résolution de l'équation caractéristique (1) permet de déterminer le nombre d'onde $k$ de l'onde de surface dans le cas du solide parfait élastique et isotrope :

$$
\begin{aligned}
& {\left[4\left(1-\varepsilon^{2} \eta^{2}\right)^{1 / 2}\left(1-\eta^{2}\right)^{1 / 2}-\left(2-\eta^{2}\right)^{2}\right]^{2}+} \\
& \quad+\left(\rho_{\mathrm{L}} / \rho\right)^{2}\left(\left(1-\varepsilon^{2} \eta^{2}\right) /\left(\delta^{2} \eta^{2}-1\right)\right) \eta^{8}=0
\end{aligned}
$$

avec : $\eta=k_{\mathrm{t}} / k, \varepsilon=k_{1} / k_{\mathrm{t}}$ et $\delta=k_{\mathrm{L}} / k_{\mathrm{t}}$

$k_{\mathrm{t}}$ : nombre d'onde transversale dans le solide;

$k_{1}$ : nombre d'onde longitudinale dans le solide;

$k_{\mathrm{L}}$ : nombre d'onde longitudinale dans le liquide ;

$\rho_{\mathrm{L}}$ : densité du liquide ;

$\rho$ : densité du solide.

Cette équation possède une racine réelle correspondant à l'onde appelée onde de Stonely [15]. Cette onde possède les caractéristiques suivantes : elle se propage parallèlement à l'interface solideliquide à une vitesse légèrement inférieure à la vitesse des ondes longitudinales dans le liquide et la majeure partie de son énergie réside dans le liquide. Cette onde n'est pas responsable des signaux que nous avons détectés, car elle ne peut être générée dans de bonnes conditions que par une onde arrivant sous incidence rasante sur l'interface liquide-solide.

L'équation (1) possède également une racine complexe correspondant à une onde qui comprend trois composantes : une se propage dans le liquide et les deux autres dans le solide. La partie imaginaire de la racine signifie que cette onde, que nous avons nommée onde de surface, mais qui est également appelée Leaky Rayleigh wave par les anglo-saxons, s'atténue suivant une loi du type $\exp (-\alpha X)$ (où $\alpha$ représente le coefficient d'atténuation) en rayonnant une onde dans le liquide. Notons que, lorsque $\rho_{\mathrm{L}}$ tend vers 0 , cette onde peut être assimilée à l'onde de Rayleigh qui se propage sans atténuation.

Bertoni et al. [17] ont calculé l'angle de réémission de cette onde et ont montré que la différence $\Delta \theta$ entre cet angle et $\theta_{\mathrm{R}}$ est très faible : $\Delta \theta \leqslant$ 10 ' suivant les matériaux.

Le résultat de l'étude angulaire du champ diffusé vers l'avant (Fig. 5) est donc en accord avec les prévisions de Bertoni et al.

Nous avons résolu l'équation (1) afin de calculer les valeurs théoriques de la vitesse $C_{\mathrm{R}}$ des ondes de surface, ainsi que celles du coefficient d'atténuation 
$\alpha$ dans le cas de nos interfaces en utilisant les valeurs suivantes $[14]$ :

$$
\begin{aligned}
& \text { - eau : } \\
& \text { - } \rho_{\mathrm{L}}=0,998 \mathrm{~g} / \mathrm{cm}^{3} \\
& \text { - } C_{\mathrm{L}}=1498 \mathrm{~m} / \mathrm{s} \quad\left(\grave{a} 25^{\circ} \mathrm{C}\right) \text {; } \\
& \text { - duralumin : } \\
& \text { - } \rho=2,79 \mathrm{~g} / \mathrm{cm}^{3} \\
& \text { - } C_{1}=6320 \mathrm{~m} / \mathrm{s} \\
& \text { - } C_{\mathrm{t}}=3130 \mathrm{~m} / \mathrm{s} \\
& \text { - acier : } \\
& \text { - } \rho=7,85 \mathrm{~g} / \mathrm{cm}^{3} \\
& \text { - } C_{1}=5960 \mathrm{~m} / \mathrm{s} \\
& \text { - } C_{\mathrm{t}}=3235 \mathrm{~m} / \mathrm{s} \text {. }
\end{aligned}
$$

Les résultats présentés dans le tableau I confirment que l'interface eau-acier est beaucoup plus propice à la propagation des ondes de surface que l'interface eau-duralumin : en effet, dans ce dernier cas, l'amplitude de l'onde est divisée par 10 lorsqu'elle a parcouru $3,8 \mathrm{~mm}$ alors qu'un parcours de $10,4 \mathrm{~mm}$ le long de l'interface eau-acier est nécessaire pour obtenir le même effet.

Tableau I. - Valeurs théoriques de la vitesse de propagation et de l'atténuation d'une onde de surface se propageant à une interface duralumin-eau ou à une interface acier-eau.

[Calculated values of the leaky Rayleigh wave velocity and attenuation at water-duraluminum and water-steel boundaries.]

\begin{tabular}{lcc}
\hline & $C_{\mathrm{R}} / C_{\mathrm{t}}$ & $\alpha \lambda$ \\
\hline Duralumin-eau & 0,937 & 0,179 \\
\hline Acier-eau & 0,926 & 0,066 \\
\hline
\end{tabular}

Rappelons que le fait que le produit $\alpha \lambda$ soit constant indique simplement que plus la fréquence de l'onde est élevée, plus l'onde s'atténue rapidement. Nous avons ainsi tracé sur le même graphique (Fig. 13) l'évolution des densités spectrales déterminées aux fréquences 3,6 et $10 \mathrm{MHz}$ (respectivement courbes b, d et f) à partir des spectres (cf. Fig. 7) et l'évolution théorique des amplitudes déduite du tableau I (respectivement courbes a, c et e). Cette présentation montre bien que les densités spectrales s'affaiblissent suivant une loi exponentielle, mais que le coefficient d'atténuation $\alpha^{\prime}$ correspondant (valeur absolue de la pente des droites de la Fig. 13) est toujours supérieur à celui $(\alpha)$ donné par la loi théorique. Cet écart $\Delta \alpha$ entre les pentes théoriques et expérimentales peut s'expliquer par le fait que les courbes théoriques ne tiennent pas compte de l'atténuation intrinsèque au matériau. Afin de vérifier ce point nous avons par ailleurs mesuré l'atténuation d'une onde de Rayleigh pure se propageant à la surface du même échantillon et pour laquelle l'atté-

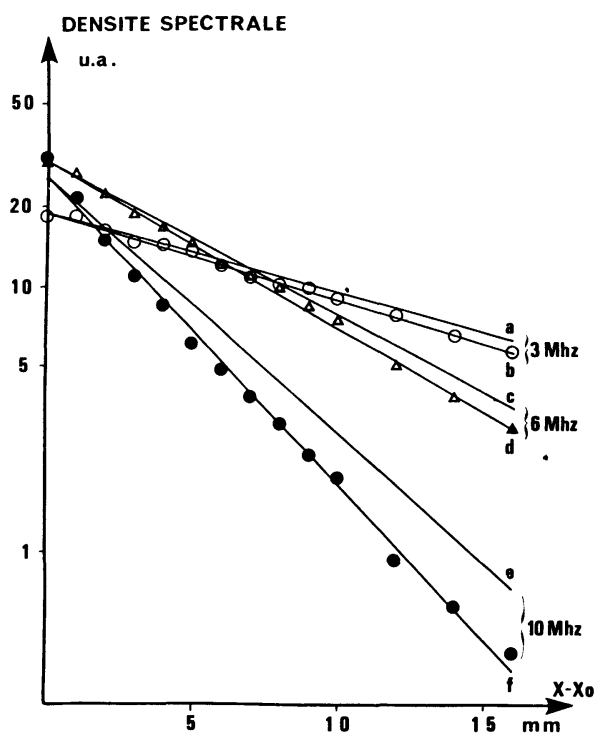

Fig. 13. - Evolution des densités spectrales en fonction du chemin $x-x_{0}$ parcouru par l'onde de surface : comparaison avec la théorie. Echelle logarithmique : (a) evolution théorique à $3 \mathrm{MHz}$; (b) évolution expérimentale à $3 \mathrm{MHz}$; (c) évolution théorique à $6 \mathrm{MHz}$; (d) évolution expérimentale à $6 \mathrm{MHz}$; (e) évolution théorique à $10 \mathrm{MHz}$; (f) évolution expérimentale à $10 \mathrm{MHz}$.

[Evolution of the spectral densities versus the distance $x-x_{0}$ travelled by the surface wave ; logarithmic scale : (a) theoretical evolution at $3 \mathrm{MHz}$; (b) experimental evolution at $3 \mathrm{MHz}$; (c) theoretical evolution at $6 \mathrm{MHz}$; (d) experimental evolution at $6 \mathrm{MHz}$; (e) theoretical evolution at $-10 \mathrm{MHz}$; (f) experimental evolution at $10 \mathrm{MHz}$.]

nuation est alors principalement due à l'atténuation de l'onde intrinsèque au matériau : ces mesures confirment l'explication avancée; en effet, les valeurs de l'atténuation $\alpha^{\prime}$ de l'onde de Rayleigh sont sensiblement égales aux écarts $\Delta \alpha$ mesurés précédemment $\quad\left(3 \mathrm{MHz}: \quad \alpha^{\prime \prime}=0,065 \pm\right.$ $0,005 \mathrm{~dB} / \mathrm{mm} \quad \Delta \alpha=0,06 \mathrm{~dB} / \mathrm{mm}, \quad 6 \mathrm{MHz}: \alpha^{\prime \prime}=$ $0,137 \pm 0,007 \mathrm{~dB} / \mathrm{mm} \quad \Delta \alpha=0,1 \mathrm{~dB} / \mathrm{mm}, 10 \mathrm{MHz}$ : $\left.\alpha^{\prime \prime}=0,39 \pm 0,05 \mathrm{~dB} / \mathrm{mm} \quad \Delta \alpha=0,39 \mathrm{~dB} / \mathrm{mm}\right)$. Autrement dit, on vérifie ainsi que l'atténuation totale $\alpha^{\prime}$ est quasiment égale à la somme de l'atténuation due aux pertes par rayonnement dans le liquide $(\alpha)$ et de l'atténuation due aux mécanismes de dispersion dans le solide $\left(\alpha^{\prime \prime}\right)$.

4.1.2 Interaction d'une onde de surface avec un défaut. - L'interaction d'une onde de Rayleigh (interface solide-vide ou gaz) avec une fissure ou une entaille a été étudiée par de nombreux auteurs. Il est possible de citer notamment les travaux de Tittmann et al. [18] ou ceux de Cooper et al. [19] ou bien encore ceux de Achenbach et al. [20].

Le but principal de ces travaux est de proposer des méthodes permettant de déterminer la profondeur d'une fissure et, par conséquent, de pouvoir donner par exemple une évaluation de la durée de vie résiduelle d'une structure. 
Plus rares sont les études consacrées à l'interaction d'une onde de surface (interface solide-liquide) avec une fissure. Bond et Saffari [11] et Fitting et al. [21] ont cependant utilisé la technique par immersion pour étudier l'interaction d'une onde de surface avec une entaille de quelques dixièmes de millimètre à quelques millimètres de profondeur.

Une schématisation de l'interaction de l'onde de surface avec l'entaille, telle que Fitting et al. [21] l'interprètent, est donnée par la figure 14. Une partie ( $r$ ) de l'onde est réfléchie au point $\mathbf{M}$ et retourne en direction du traducteur qui la détecte. Ils ont également mis en évidence un deuxième signal (s) : dans le cas où la fissure est remplie d'air (Fig. 14a) celui-ci provient de la réflexion au point $\mathrm{T}$ de l'onde de Rayleigh transmise le long de l'entaille. Lorsque l'entaille est remplie d'eau (Fig. 14b) ce

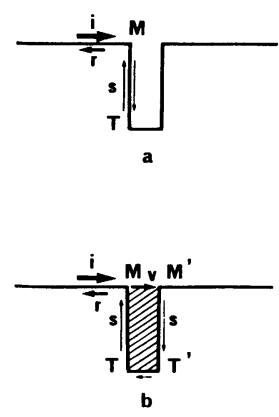

Fig. 14. - Interaction d'une onde de surface avec une entaille d'après Fitting et al. [21]: i : onde de surface incidente; $\mathrm{r}$ : onde de surface réfléchie; $\mathrm{s}$ : onde de surface transmise le long de l'entaille $; \mathrm{v}$ : onde transmise dans le liquide. (a) Défaut contenant de l'air ; (b) défaut contenant de l'eau.

[Surface wave interaction with a notch (from Fitting et al. [21]) : $\mathrm{i}$ : incident surface wave; $\mathrm{r}$ : reflected surface wave; $\mathrm{s}$ : surface wave transmitted along the notch; $\mathrm{v}$ : bulk wave transmitted to the liquid. (a) Defect filled with air ; (b) defect filled with water.]

deuxième signal est retardé dans le temps; les auteurs considèrent que l'onde de surface incidente au point $M$ est convertie en partie en onde de volume (v) se propageant dans le liquide jusqu'à $M^{\prime}$ où elle se reconvertit alors en une onde de surface (s) parcourant le trajet $\mathbf{M}^{\prime} \mathrm{T}^{\prime} \mathrm{TM}$. Précisons dès maintenant que, dans le cadre de nos expériences, ce deuxième signal n'a pas été étudié car, aussi bien dans le cas de la fissure réelle que dans celui du défaut modèle, le trajet MT représente plusieurs centimètres : l'onde qui est transmise le long de cette face est entièrement atténuée avant de revenir au point $\mathrm{M}$.

Par ailleurs, Fahr et al. [10] donnent une interprétation sensiblement équivalente de l'interaction d'une onde de surface avec une fissure débouchante. Ces auteurs indiquent cependant la création possible d'ondes de volume diffractées, dans le liquide et dans le solide, par les bords externes et le sommet de la fissure. Ils considèrent que la présence de ces ondes, et en particulier de l'onde diffractée dans le liquide, a une influence négligeable sur le signal détecté provenant du défaut.

Compte tenu de ces quelques éléments bibliographiques et au vu de nos résutats, l'interaction entre l'onde de surface et notre défaut modèle simplifié donnerait donc lieu aux mécanismes schématisés sur la figure 15 : une partie de l'onde de surface incidente $S_{1}$ se réfléchit sur la discontinuité et repart dans la direction $\mathrm{OX}\left(\mathrm{S}_{2}\right)$; une autre partie de l'onde est diffractée sur l'arête sous la forme d'une onde de volume $\mathrm{D}$ émise dans le liquide ; enfin, et bien que ces composantes n'interviennent pas dans notre étude à cause de la grande profondeur de l'échantillon et de nos défauts modèles, la génération d'une onde de surface $\mathrm{S}_{3}$ le long du plan de la discontinuité ni celle d'ondes de volume $\mathrm{V}$ dans le solide ne doit être exclue.

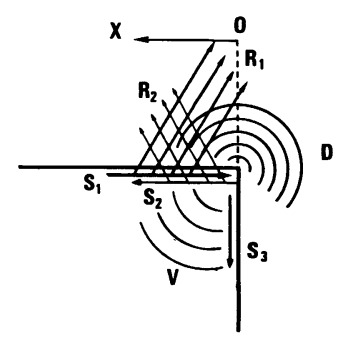

Fig. 15. - Interaction d'une onde de surface avec un défaut simplifié d'après nos résultats $: \mathrm{S}$ : ondes de surface ; $S_{1}$ : incidente; $S_{2}$ : réfléchie; $S_{3}$ : transmise; $R$ : ondes rayonnées : $R_{1}$ par $S_{1}, R_{2}$ par $S_{2} ; D$ : onde diffractée dans le liquide par l'arête; $v$ : ondes longitudinales et transversales diffractées dans le solide.

[Surface wave interaction with an idealized defect from our results : $S_{1}$ : incident surface wave $; S_{2}$ : reflected surface wave $; S_{3}$ : transmitted surface wave $; R_{1}$ : radiated wave by $S_{1} ; R_{2}$ : radiated wave by $S_{2} ; D$ : wave diffracted in the liquid; $\mathrm{v}$ : compressional and transverses waves diffracted in the solid.]

Ainsi, ce schéma permet d'interpréter les observations rapportées au paragraphe 2.2.3. En particulier, on peut expliquer l'existence de deux signaux captés distinctement dans le cas où $\theta_{\mathrm{r}}$ est négatif : Ia correspondrait à l'onde $\mathrm{D}$ et IIa correspondrait à l'onde $\mathrm{R}_{1}$ rayonnée vers l'avant par $\mathrm{S}_{1}$; on comprend alors aisément que :

- compte tenu des caractéristiques du rayonnement des ondes de surface, la composante $R_{1}$ présente une amplitude maximale en fonction de $\theta_{\mathrm{r}}$ pour un angle égal à $-\theta_{\mathrm{R}}$, conduisant à une amplitude maximale du signal global pour cette valeur (Fig. 9b) ;

- en fonction de la position $X$ qui définit l'intersection de l'axe du traducteur avec l'interface: 
l'amplitude de la composante $\mathbf{R}_{1}$ est nulle pour $X<0$ et augmentera graduellement pour $X>0$, car le rayonnement de $S_{1}$ s'effectue le long du trajet de cette onde de surface : c'est bien ce qui est observé pour la composante IIa sur la figure 10a; au contraire, la composante D n'est émise qu'au voisinage immédiat de l'arête. Cette composante donne donc lieu à un signal d'amplitude maximale lorsque l'axe du traducteur passe au voisinage de l'arête (signal Ia, Fig. 10) ;

- de plus, par les mêmes arguments que les précédents (émission localisée pour $D$ et étendue pour $\mathrm{R}_{1}$ ), on comprend que le signal Ia conserve une position temporelle fixe alors que celle de IIa évolue en fonction de l'angle $\theta_{\mathrm{r}}$ jusqu'à se superposer au signal I ;

- enfin, la détection d'un seul signal dans le domaine $b$ s'explique, d'une part, par l'importance de l'onde $R_{1}$ au voisinage de l'angle de Rayleigh et, d'autre part, par la proximité temporelle des signaux associés à $\mathrm{R}_{1}$ et $\mathrm{D}$ comme le suggère l'inversion de la position temporelle de ces deux signaux entre les domaines a et $c$ encadrant le domaine $b$.

Par ailleurs, dans le cas où $\theta_{\mathrm{r}}$ est positif, une argumentation similaire à la précédente peut être développée, mais en considérant maintenant le rayonnement $R_{2}$ de l'onde $S_{2}$ réfléchie au lieu du rayonnement $R_{1}$ qui est produit par l'onde $S_{1}$ incidente. Bien que, dans ce cas, nous n'ayons pu observer distinctement les deux composantes associées à $R_{2}$ et $D$, l'existence de cette dernière pour les angles $\theta_{\mathrm{r}}$ positifs est suggérée par la comparaison du diagramme angulaire caractérisant le rayonnement d'une onde de surface (Fig. 5b) avec celui provenant de l'arête de la discontinuité : cette comparaison est présentée sur la figure 16 pour laquelle nous avons, aussi bien pour les angles positifs que pour les angles négatifs, ajusté arbitrairement l'amplitude du maximum de rayonnement de l'onde de surface à celle du maximum global résultant de l'interaction avec la discontinuité : dans les deux cas, la différence entre les deux diagrammes ne peut s'expliquer que par l'existence d'une deuxième composante qui serait due à l'onde $\mathrm{D}$ diffractée directement dans le liquide.

Cette interprétation corrobore donc bien l'hypothèse faite par Fahr et al. [10] suivant laquelle l'onde diffractée dans le liquide affecte peu l'écho détecté lorsque l'on travaille en émission-réception à l'angle de Rayleigh. Par contre, il n'est plus possible de négliger cette composante diffractée directement dans le liquide lorsque l'angle de réception est différent de $\theta_{\mathrm{R}}$.

4.2 CARTographie D'UnE FISSURE RÉELLE. - La discussion précédente nous permet maintenant de préciser le mécanisme conduisant à la réalisation des cartographies : les composantes du faisceau focalisé
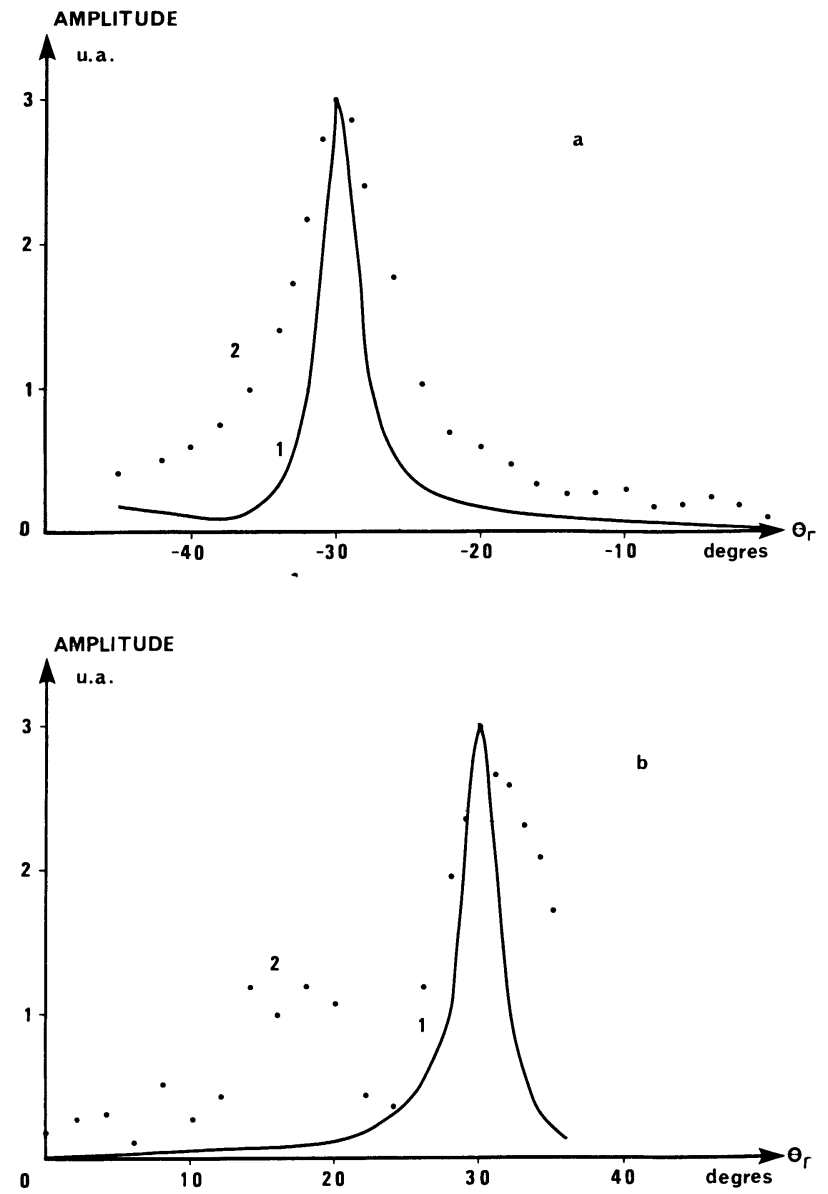

Fig. 16. - Comparaison entre les diagrammes angulaires du champ rayonné vers l'avant par une onde de surface (1) et du signal provenant du défaut simplifié (2) : (a) $-45^{\circ} \leqslant \theta_{\mathrm{r}} \leqslant 0^{\circ} ;$ (b) $0^{\circ} \leqslant \theta_{\mathrm{r}} \leqslant 36^{\circ}$. L'amplitude du spectre angulaire (2) a été ajustée à celle de (1) au niveau du maximum correspondant à $\theta_{\mathrm{r}}=-\theta_{\mathrm{R}}\left(\right.$ cas a) et $\theta_{\mathrm{r}}=$ $\theta_{\mathrm{R}}$ (cas b).

[Comparison between the angular dependence of the wave radiated by a surface wave (1) and the angular dependence of the signal due to the defect (2): (a) $-45^{\circ} \leqslant \theta_{\mathrm{r}} \leqslant$ $0^{\circ}$; (b) $0^{\circ} \leqslant \theta_{\mathrm{r}} \leqslant 36^{\circ}$. The amplitude of (2) has been normalized such that its maximum coincides with that one of (1) (at $\theta_{\mathrm{r}}=-\theta_{\mathrm{R}}$ in case a and $\theta_{\mathrm{r}}=+\theta_{\mathrm{R}}$ in case b).]

incident atteignant la surface sous l'angle de Rayleigh génèrent une onde de surface qui interagit avec la fissure. Il résulte de cette interaction une onde de surface réfléchie qui rayonne alors dans le liquide quasiment dans la direction d'incidence initiale : cette onde constitue probablement la composante principale du signal d'écho reçu par le traducteur émetteur-récepteur. Comme nous l'avons montré précédemment, une composante secondaire, associée à une onde diffractée directement dans le liquide, se superpose probablement à cette composante principale. Par ailleurs, lorsque la tache focale s'éloigne de l'arête de la fissure, l'atténuation des 
ondes de surface conduit à une décroissance progressive de l'amplitude de l'écho reçu : bien que cette décroissance ne soit pas encore assez rapide pour obtenir une résolution de l'ordre de la largeur de la tache focale du traducteur utilisé (environ $0,6 \mathrm{~mm}$ ), on comprend maintenant notre choix du couple duralumin-eau, pour la réalisation de la cartographie, choix qui a été effectué afin d'obtenir une atténuation importante bien qu'encore insuffisante des ondes de surface dans le cas de ce couple (cf. Tab. I).

A partir de ces considérations fondamentales, nous discutons maintenant deux points complémentaires. Le premier concerne l'étude angulaire du signal détecté en émission-réception qui a montré que l'amplitude de ce signal est maximale pour $\boldsymbol{\theta}_{\text {incident }}=27^{\circ}$, les cartographies ayant donc été réalisées avec le traducteur focalisé réglé sur cet angle d'incidence. Or, cet angle est différent de l'angle théorique de Rayleigh pour une interface duralumin-eau : $\theta_{\mathrm{R}}=30^{\circ} 43^{\prime}$. Compte tenu des résultats précédents où nous avons montré que l'amplitude détectée est maximale pour $\theta_{\mathrm{r}}=\boldsymbol{\theta}_{\mathrm{R}}$, il serait logique de penser que le meilleur angle d'émission-réception soit égal à $\theta_{R}$. Au stade actuel de ce travail, deux hypothèses peuvent être avancées pour expliquer cette différence : la première est que le rendement optimum du traducteur focalisé pour la production des ondes de surface et la détection du faisceau rayonné en retour n'est pas obtenue dans les mêmes conditions que celui d'un traducteur-plan. La seconde est que la localisation de la tache focale au voisinage de l'arête de la fissure pourrait conduire à prendre en compte l'émoussement local de cette arête dans les mécanismes discutés précédemment.

Le deuxième point que nous commentons concerne la présence d'importantes variations d'amplitude le long de la trace de la fissure. Celles-ci peuvent être attribuées aux désorientations locales du plan de fissure vis-à-vis de la direction de l'onde incidente (cf. Fig. 2b). En effet, nous avons montré la forte dépendance de l'amplitude détectée en fonction de l'orientation du plan d'incidence
(Fig. 12). Ainsi, cette amplitude diminue de $75 \%$ pour un angle de désorientation égal à $10^{\circ}$.

\section{Conclusion.}

Au cours de ce travail, nous avons étudié l'origine des mécanismes conduisant à la cartographie de la trace externe de défauts débouchants réalisée avec un appareillage ultrasonore de type $C$ Scan.

Après une première partie dans laquelle nous avons étudié expérimentalement la propagation des ondes de surface, nous avons analysé l'interaction de celles-ci avec un défaut modèle. Nous avons alors montré que le mécanisme suivant serait responsable des échos exploités pour la cartographie :

Les composantes du faisceau focalisé incident frappant la surface sous l'angle de Rayleigh $\theta_{R}$ génèrent une onde de surface qui se réfléchit en partie sur le défaut. Cette onde réfléchie rayonne alors en retour une onde dirigée principalement dans la direction définie par l'angle $\theta_{R}$. De plus, nous avons mis en évidence une deuxième onde, moins importante et moins directive que cette composante principale, qui contribuerait au signal détecté et qui serait dû à la diffraction dans le liquide de l'onde de surface par l'arête du défaut.

Dans la suite, ce travail devra être complété par une étude de l'influence, sur les phénomènes observés, de l'ouverture d'une fissure réelle. L'application de ce phénomène à la cartographie des défauts acoustiques est encore limitée par la faible résolution latérale qui résủlte de l'atténuation insuffisante des ondes de surface mises en jeu; néanmoins, cette résolution est sensiblement améliorée en travaillant à plus haute fréquence, l'atténuation des ondes de surface augmentant linéairement avec la fréquence.

\section{Remerciements.}

Nous remercions vivement J. C. Baboux et l'équipe du Professeur M. Perdrix qui ont contribué à l'analyse spectrale des signaux.

\section{Bibliographie}

[1] Chicois, J., Fougeres, R., Guichon, G., Hamel, A. and Vincent, A., Acta Metall. 34 (1986) 2157-2170.

[2] Joshi, N. R. and Green, R. E., Mater. Eval. (February, 1975) 25-29, 36.

[3] BouAmi, D. et DE VAdder, D., Mémoires et Etudes Scientifiques, Rev. Métall. (mai, 1986) 249-257.

[4] Adler, L., Research Techniques in Nondestructive testing (Academic press, London) III (1977) 149.
[5] SILK, M. G., Research techniques in Nondestructive Testing (Academic press, London) III (1977) 5199.

[6] Burger, C. P. and Testa, A., Proc. Symp. Nondestructive Eval., 13th (San Antonio) April, 1981, pp. 9-18.

[7] Tien, J. J., Khuri-YaKub, B. T., Kino, G. S., Evans, A. G. and Marshall, D. B., Review of progress in quantitative Nondestructive evaluation (Plenum Press, New York) 1982, pp. 569-571. 
[8] Khuri-Yakub, B. T., Kino, G. S., Liang, K., Tien, J. J., Chou, C. H., Evans, A. G. and MARSHALL, D. B., Review of progress in quantitative Nondestructive evaluation (Plenum Press, New York) 1982, pp. 601-605.

[9] Khuri-Yakub, B. T., Shui, Y., Kino, G. S., MarsHALl, D. B. and Evans, A. G., Review of progress in quantitative NDE (Santa Cruz) August, 1983, pp. 229-237.

[10] Fahr, A., Johar, S., Murthy, M. K. and SturROCK, W. K., Review of progress in quantitative NDE (Santa Cruz) August, 1983, pp. 239-249.

[11] BOND, L. J., SAFFARI, N., Review of progress in quantitative NDE (Santa Cruz) August, 1983, pp. 251-262.

[12] Yamanika, K. and Enomoto, Y., J. Appl. Phys. 53 (1982) 846-850.

[13] Ge, Y. J. and Vincent, A., J. Phys. E 20 (1987) 1019-1025.

[14] Handbook of Chemistry and Physics, 67th (The Chemical Rubber Co, Cleveland).
[15] Viktorov, I. A., Rayleigh and Lamb waves (Plenum press, New York) 1967.

[16] BREKHOVSKIKH, M., Waves in layered media (Academic press, New York) 1973.

[17] Bertoni, H. L. and TAmir, T., J. Appl. Phys. 2 (1973) $157-172$.

[18] Tittmann, B. R. and Buck, O., J. Nondestr. Eval. 1 (1980) 123-136.

[19] Cooper, J. A., Crosbie, R. A., Dewhurst, R. J., MCKIE, A. D. W. and PAlmer, S. B., IEEE Trans. Ultrason., Ferroelectrics and Frequency Control UFFC-33 (1986) 462-470.

[20] Achenbach, J. D., Gautesen, A. K. and MendelSHON, D. A., IEEE Trans. Sonics and Ultrason. SU-27 (1980) 124-129.

[21] Fitting, D., Adler, L. and De Billy, M., Review of progress in quantitative Nondestructive evaluation (Plenum press, New York) 1982, pp. 525-534. 